\title{
VARIATIONS OF HODGE STRUCTURE, LEGENDRE SUBMANIFOLDS, AND ACCESSIBILITY
}

\author{
JAMES A. CARLSON AND DOMINGO TOLEDO
}

\begin{abstract}
Variations of Hodge structure of weight two are integral manifolds for a distribution in the tangent bundle of a period domain. This distribution has dimension $h^{2,0} h^{1,1}$ and is nonintegrable for $h^{2,0}>1$. In this case it is known that the dimension of an integral manifold does not exceed $\frac{1}{2} h^{2,0} h^{1,1}$. Here we give a new proof, based on an analogy between Griffiths' horizontal differential system of algebraic geometry and the contact system of classical mechanics. We show also that any two points in such a domain can be joined by a horizontal curve which is piecewise holomorphic.
\end{abstract}

\section{INTRODUCTION}

A problem which goes back to Pfaff, Frobenius, and E. Cartan is that of finding and characterizing the integral manifolds of a differential system. Of particular interest are homogeneous nonintegrable systems on a homogeneous space. The focus of this note is a subclass of these which arise in algebraic geometry: Griffith horizontal differential systems [20, Definition 25], integral manifolds of which are usually known as variations of Hodge structure (pure or mixed).

Our main goal is to isolate in easily understood cases what we believe to be general characteristics of the Griffiths systems. Among these are: (a) that the dimension of integral manifolds is usually about one half the dimension of the horizontal distribution, provided that this latter is nonintegrable, and (b) that integral manifolds of maximal dimension are generally rigid in the sense that they are conjugate under an element of the group acting on the underlying space. In addition, it appears that (c) integral manifolds of maximal dimension are usually degenerate in a specific way, and that (d) nondegenerate manifolds tend to satisfy much stricter dimension bounds than in the general case. Accordingly, there may be integral manifolds which are maximal, but not of maximal dimension. Here, to say that an integral manifold is maximal is to say that it is not contained in an integral manifold of strictly larger dimension. Thus, we also (e) seek criteria to recognize when integral manifolds are maximal. Finally, we remark that all of the systems to be considered below satisfy (f) the

Received by the editors November 11, 1987.

1980 Mathematics Subject Classification (1985 Revision). Primary 14C30.

Research partially supported by the National Science Foundation. 
accessibility condition. By this we mean that any two points of the homogeneous space can be connected by a piecewise holomorphic integral curve. This is, of course, impossible for integrable systems, since the two points may lie in different leaves.

The special feature of the Griffiths systems that we exploit is that formally they resemble those of a matrix-valued contact form. Therefore the first example which we study is the complex form of the classical contact system, given on $D=\mathbb{C}^{2 n+1}$ by the differential form

$$
\omega=d r-\sum_{i=1}^{n} p_{i} d q_{i}
$$

Integral manifolds are of dimension at most $n$, and those of maximal dimension are called Legendre manifolds [1, Appendix 4]. The contact system is homogeneous relative to a natural action of the complex Heisenberg group, and as such it may be identified as a classifying space for mixed Hodge structures. The Legendre manifolds are therefore variations of Hodge structure of maximal dimension.

To exploit the analogy with contact geometry, we introduce canonical coordinates in the period domains, given by exponentiating a natural nilpotent subalgebra of $\mathfrak{g}_{\mathbb{C}}$, the complexified Lie algebra of the isometry group. The local nil-homogeneous structure that this imposes mirrors the global nil-homogeneous structure of the contact system. For weight two domains the resulting equations for the differential system are rather easy to analyze, and from them we obtain the dimension bound.

Note that for weight one domains there is nothing interesting to say, since the differential system is trivial. A deeper analysis of the matrix-valued forms given by the method employed below may yield the correct bounds in higher weight.

The plan of the paper is as follows. We first discuss some generalities on differential systems and then study two model cases: the contact system and what we shall call the simple Tate system, both of which are defined on a nil-manifold. Next, we recall some basic facts about the geometry of period domains, after which we investigate integral manifolds in weight two domains. In particular, we give a new proof of the following result [2, Theorem 1.1], sharp for $h^{1,1}$ even by [2, Theorem 6.4]. ${ }^{1}$

(1.1) Theorem. Let $D$ be a weight two period domain with $h^{2,0}>1$. Then any integral submanifold $S$ satisfies $\operatorname{dim} S \leq \frac{1}{2} h^{2,0} h^{1,1}$.

In this case the horizontal distribution has dimension $h^{2,0} h^{1,1}$, so that variations of weight two structures can have dimension no greater than half that of the distribution to which they are tangent. Note that in the case of contact

${ }^{1}$ Using different methods the authors have recently obtained results which give an improved (and sharp) bound for $h^{1,1}$ odd: $\operatorname{dim} S \leq \frac{1}{2} h^{2,0}\left(h^{1,1}-1\right)+1$. 
manifolds, the maximum values of the ratio 'dimension of integral manifold to dimension of distribution' is also about one-half. As stated above, we believe this to be a general phenomenon [2, p. 60, line 9]. The results of $\S 4$ are an indication of this, as are the examples of [9, Remark 2]. For nondegenerate variations of higher weight one has the bounds of [2, Theorems 4.1, 4.2]. ${ }^{2}$

For all of the cases considered below we establish the accessibility property, essentially a corollary of a theorem of Chow [11]. We do, however, give an independent argument for the weight two case, one motivated by similar but simpler considerations for the contact system. The accessibility problem has been studied in the context of control theory [21, 22].

\section{DifFERENTIAL SYSTEMS}

Let us now review some basic notions in the theory of differential systems. To this end, let $T^{*}$ denote the cotangent sheaf of a manifold $M$-real, complex, or holomorphic, according to the context-and let $\Lambda^{*} T^{*}$ be the associated deRham algebra. A differential system is a differential ideal in the deRham algebra, i.e., an ideal $\mathscr{I}$ which is closed under exterior differentiation. An integral manifold for $\mathscr{I}$ is a mapping $i: S \rightarrow M$ such that $i^{*} \omega=0$ for each germ $\omega$ of $\mathscr{I}$. Note that $i^{*} d \omega=0$ as well. Often we take $i$ to be an inclusion, so that $S$ is a submanifold; in any case, when we speak of the dimension of an integral manifold, we speak of the dimension of $i(S)$.

A differential system is Pfaffian if $\mathscr{I}$ is generated by germs of 1 -forms. We shall denote the stalk of $\mathscr{I}$ at $x$ by $\mathscr{F}(x)$ and the fiber-the quotient by $m(x) \mathscr{I}(x)$, where $m(x)$ is the maximal ideal in the structure sheaf-by $\mathscr{J}_{x}$. The dimension of the system is that of $\mathscr{F}_{x}^{1}$, that is, of the degree one part of the fiber. In all that follows, we shall assume that the pointwise dimension of a Pfaffian system is constant, so that we may speak of the dimension of $\mathscr{I}$. Dual to such a system is the associated distribution: the field of tangent subspaces $x \mapsto \mathscr{D}_{x}$, where $\mathscr{D}_{x}$ is the space of tangent vectors annihilated by $\mathscr{F}_{x}$. In this language an integral manifold for $\mathscr{I}$ is one whose tangent space at $x$ is a subspace of $\mathscr{D}_{x}$ for all $x$. Clearly, one has $\operatorname{dim} \mathscr{D}+\operatorname{dim} \mathscr{J}=\operatorname{dim} M$ as well as

$$
\operatorname{dim} S \leq \operatorname{dim} \mathscr{D}
$$

for all integral manifolds $S$.

A Pfaffian system is integrable if its stalks are generated by closed 1-forms $\phi_{1}, \ldots, \phi_{k}$. On a sufficiently small open set we may assume that the $\phi$ 's are independent, so that functions $f_{i}$ with $d f_{i}=\phi_{i}$ exist and give a partial local coordinate system. All integral manifolds then have local defining equations $f(x)=c_{i}$ for some constants $c_{i}$, as a result of which equality holds in (2.1).

To obtain a computationally useful criterion, consider a Pfaffian system $\mathscr{J}$ with minimal local generating set $\Phi=\left\{\phi_{1}, \ldots, \phi_{k}\right\}$. Then $\mathscr{J}$ is integrable if

\footnotetext{
${ }^{2}$ See [2, Erratum].
} 
and only if there exist one-forms $\alpha_{i j}$ such that $d \phi_{i}=\sum \alpha_{i j} \wedge \phi_{j}$. In other words, the differential and algebraic ideals generated by $\Phi$ coincide. Equivalently, $\mathscr{I}$ is integrable if

$$
d \phi_{i} \wedge \phi_{1} \wedge \cdots \wedge \phi_{k}=0 \text { for all } i
$$

Dual to these is Frobenius' criterion: $\mathscr{D}$ is integrable if the Lie bracket of vector fields belonging to $\mathscr{D}$ again belongs to $\mathscr{D}$. A vector field belongs to $\mathscr{D}$ if $\xi_{x} \in \mathscr{D}_{x}$ for all $x$.

Once a system is recognized as nonintegrable, one is faced with the problem of bounding the dimension of its integral manifolds. As we shall see in a moment, the following often gives excellent results.

(2.3) Lemma. Let $U$ be an open set in $\mathbb{C}^{n}$ and let $\mathscr{I}$ be a differential ideal in the cotangent algebra of $U$. Suppose that a local volume form $\Omega=f(z) d z_{1} \wedge$ $\cdots \wedge d z_{n}$ can be written as

$$
\Omega=\left(\phi_{1} \wedge \cdots \wedge \phi_{p}\right) \wedge\left(\psi_{1} \wedge \cdots \wedge \psi_{q}\right) \wedge \alpha(a),
$$

where $\phi_{i} \in \mathscr{I}^{1}, \psi_{j} \in \mathscr{I}^{2}$, and $\alpha(a)$ is an arbitrary form of degree a. If $S$ is an integral manifold for $\mathscr{I}$ then $\operatorname{dim} S \leq q+a$.

Proof. Suppose that $\operatorname{dim} S=d$, let $\tau_{1}, \ldots, \tau_{d}$ be a basis for the tangent space to $S$ at some point, and let $\nu_{1}, \ldots, \nu_{e}$ be the set of 'normal' vectors which complete the set of $\tau$ 's to a basis for the tangent space to $U$. One the one hand we have

$$
\Omega\left(\tau_{1}, \ldots, \tau_{d}, \nu_{1}, \ldots, \nu_{e}\right) \neq 0,
$$

since $\Omega$ is a volume form. On the other hand,

$$
\begin{aligned}
& \Omega\left(\tau_{1}, \ldots, \tau_{d}, \nu_{1}, \ldots, \nu_{e}\right) \\
& \quad=\left[\left(\phi_{1} \wedge \cdots \wedge \phi_{p}\right) \wedge\left(\psi_{1} \wedge \cdots \wedge \psi_{q}\right) \wedge \alpha\right]\left(\tau_{1}, \ldots, \tau_{d}, \nu_{1}, \ldots, \nu_{e}\right) .
\end{aligned}
$$

The right-hand side is a sum of terms of the form

$$
\phi_{1}\left(t_{1}\right) \cdots \phi_{p}\left(t_{p}\right) \psi_{1}\left(u_{1}, v_{1}\right) \cdots \psi_{q}\left(u_{q}, v_{q}\right) \alpha\left(w_{1}, \ldots, w_{a}\right)
$$

where the ordered set $\left(t_{1}, \ldots, t_{p}, u_{1}, v_{1}, \ldots, u_{q}, v_{q}, w_{1}, \ldots, w_{a}\right)$ is a permutation of $\left(\tau_{1}, \ldots, \tau_{d}, \nu_{1}, \ldots, \nu_{e}\right)$. Such a term will vanish unless

(i) none of the $t_{i}$ are tangent,

(ii) at most one of each pair $\left(u_{i}, v_{i}\right)$ is tangent.

The existence of a nonvanishing term then implies that there are no more than $q+a$ tangent vectors, as required. More generally, one can assert the following:

(2.4) Lemma. Let $U$ be an open set in $\mathbb{C}^{n}$ and let $\mathscr{I}$ be a differential ideal in the cotangent algebra of $U$. Suppose that a local volume form $\Omega=f(z) d z_{1} \wedge$ $\cdots \wedge d z_{n}$ can be written as

$$
\Omega=\phi\left(1, r_{1}\right) \wedge \cdots \wedge \phi\left(k, r_{k}\right) \wedge \alpha(a)
$$


where $\phi\left(p, r_{p}\right)$ is a product of $r_{p}$ elements of $\mathscr{J}^{p}$ and $\alpha(a)$ is an arbitrary form of degree $a$. Then

$$
\operatorname{dim} S \leq a+\sum_{p=2}^{k} r_{p}(p-1)
$$

for any integral manifold $S$ of $\mathscr{I}$.

Proof. Similar to the above.

To obtain the best possible estimates, one should maximize the number of low degree forms in the differential ideal which appear in the factorization of the volume form.

Finally, we consider the accessibility problem for nonintegrable systems which we touched upon in the introduction. The basic result is one of Chow [11], which we restate here for complex manifolds:

(2.5) Theorem (Chow). Let $U$ be an open set in $\mathbb{C}^{n}$, let $\mathscr{D}$ be a distribution in the holomorphic tangent bundle of $U$ generated by global vector fields, and suppose that $\mathscr{D}$ generates the tangent bundle under the Lie bracket. Then any two points of $U$ are accessible by a piecewise holomorphic curve tangent to $\mathscr{D}$.

\section{THE CONTACT SYSTEM}

The contact system of genus $n$, with differential ideal generated by a single one-form

$$
\omega=d r-\sum_{i=1}^{n} p_{i} d q_{i}=d r-p \cdot d q
$$

in coordinates $(p, q, r)=\left(p_{1}, \ldots, p_{n}, q_{1}, \ldots, q_{n}, r\right)$, is the prototypical nonintegrable system. Its behavior, which we will study in the light of the preceding discussion, models much of what we shall encounter in more complicated situations.

First, note that a volume element can be written as a product of forms in the contact ideal:

$$
\omega \wedge(d \omega)^{n}= \pm d \text { Vol }
$$

where $d \mathrm{Vol}=d r \wedge d p_{1} \wedge \cdots \wedge d p_{n} \wedge d q_{1} \wedge \cdots \wedge d q_{n}$. Therefore $d \omega \wedge \omega \neq 0$, and so, by (2.2), the contact system is indeed nonintegrable.

Since this system is defined by a single one-form, the associated distribution is of codimension 1, i.e., of dimension $2 n$. Its integral manifolds, however, are small:

(3.3) Proposition. The dimension of a contact manifold of genus $n$ is not most $n$.

The proof follows immediately from Lemma (2.3) and the expression (3.2) above for the volume element as a product of forms in the contact ideal. 
Integral manifolds of dimension $n$ exist. For a local solution, choose a 'generating function' $f: W \rightarrow \mathbb{C}$ where $W$ is open in $\mathbb{C}^{n}$, and set $r=f(q)$, $p=\nabla f$. According to [1, Appendix 4], all Legendre manifolds can be written in this form locally, up to possible exchange of $p$ 's and $q$ 's.

The contact system is homogeneous. To see this, consider the complex Heisenberg group of genus $n$. This is the group $G_{n}$ of the unipotent matrices

$$
U(p, q, r)=\left(\begin{array}{ccccccc}
1 & & & & & \\
q_{1} & 1 & & & & & \\
\cdot & 0 & \cdot & & & & \\
\cdot & \cdot & & \cdot & & & \\
\cdot & \cdot & & & \cdot & & \\
q_{n} & 0 & \cdot & \cdot & 0 & 1 & \\
r & p_{1} & \cdot & \cdot & \cdot & p_{n} & 1
\end{array}\right),
$$

which we shall often abbreviate as

$$
U=\left(\begin{array}{lll}
1 & 0 & 0 \\
q & 1 & 0 \\
r & p & 1
\end{array}\right)
$$

Then the components of the Maurer-Cartan matrix

$$
\Omega=U^{-1} d U=\left(\begin{array}{ccc}
0 & 0 & 0 \\
d q & 0 & 0 \\
\omega & d p & 0
\end{array}\right)
$$

are left-invariant, and $\omega=d r-p \cdot d q$ is the contact form. Identifying $G_{n}$ with $\mathbb{C}^{2 n+1}$, we achieve the asserted homogeneity.

The contact system is also a special case of Griffiths' horizontal distribution, whose definition we now recall. Let $F^{*}=\left[\cdots \supset F^{p} \supset F^{p+1} \supset \cdots\right]$ be a family of filtrations on a complex vector space $H_{\mathbb{C}}$ which is holomorphically parametrized by a complex manifold $M$. Filtrations satisfying

$$
\partial F^{p} / \partial z_{i} \subset F^{p-1}
$$

where $z_{i}$ is part of a local coordinate system, are called horizontal. Objects of this kind arise naturally in algebraic geometry as variations of Hodge structure, both pure and mixed [12,15-18,20,23]. To write the above condition in the language of differential systems, choose a local holomorphic frame $\left\{\alpha_{i} \mid i=\right.$ $1, \ldots, n\}$ for $H_{\mathbb{C}}$ such that $\left\{\alpha_{i} \mid i=1, \ldots, n\right\}$ is a frame for $F^{p}$, where $n_{p} \geq n_{p+1}$. Set $\beta_{p}=\alpha_{1} \wedge \cdots \wedge \alpha_{n_{p}}$, and let $I_{p}=\left\{i \mid n_{p} \geq i>n_{p+1}\right\}$ be the set of indices corresponding to the vector-valued functions which frame $F^{p}$ modulo $F^{p+1}$. Then the equations

$$
d \alpha_{i} \wedge \beta_{p-1}=0
$$

for $i \in I_{p}$ define the horizontal system. 
To identify the contact and horizontal systems, let $e_{0}, e_{1}, \ldots, e_{n}, e_{n+1}$ be the standard basis for $\mathbb{C}^{n+2}$, and define a filtration $F(p, q, r)$ by

$$
\begin{aligned}
& F^{0}=\text { span of }\left\{\alpha_{0}=e_{0}+\sum_{i=1}^{n} q_{i} e_{i}+r e_{n+1}\right\}, \\
& F^{-1}=F^{0}+\text { span of }\left\{\alpha_{i}=e_{i}+p_{i} e_{n+1} \mid i=1, \ldots, n\right\}, \\
& F^{-2}=\mathbb{C}^{n+2} .
\end{aligned}
$$

The Heisenberg group acts transitively and effectively on the set $D$ of all such filtrations, with

$$
F(p, q, r)=U(p, q, r) F(0,0,0) .
$$

Therefore the complex manifolds $D$ and $G$ may be identified, and, via this identification, the contact form may be transferred to $D$. Now apply the prescription of (3.5) to compute the horizontal system:

$$
d \alpha_{0} \wedge \beta_{-1}=d \alpha_{0} \wedge \alpha_{0} \wedge \alpha_{1} \wedge \cdots \wedge \alpha_{n}= \pm(d r-p \cdot d q) e_{0} \wedge \cdots \wedge e_{n+1} .
$$

The coefficient one-form which appears on the right-hand side is the contact form, as claimed.

(3.6) Remark. The space of filtrations $D$ is a classifying space for mixed Hodge structures $H$ whose graded quotients are Hodge structures of type $(p, p)$ for $p=0,-1,-2$. More specifically, $\mathrm{Gr}^{W} H \cong \mathbb{Z}(0) \oplus \mathbb{Z}(1)^{n} \oplus \mathbb{Z}(2)$, where the $\mathbb{Z}(p)$ are Tate structures. As we show in the appendix, these 'Heisenberg variations' occur geometrically: there is a 4-dimensional Legendre manifold $S$ defined by the period mapping for a configuration of lines in $\mathbb{P}^{2}$.

By a Tate structure $\mathbb{Z}(p)$ one means the Hodge structure with $H_{\mathbb{Q}}=\mathbb{Q}$, $H_{\mathbb{Z}}=(2 \pi i)^{p} \mathbb{Z} \subset H_{\mathbb{C}}=\mathbb{C}$, with $H_{\mathbb{C}}$ of pure type $(-p,-p)$. For the geometric example, see $\S 10$.

Finally, let us consider the accessibility problem. Since $D \cong G$ is homogeneous, it suffices to verify Chow's condition at the origin, which we take to be the identity element of the Lie group. The tangent space there is given by the Lie algebra, i.e., by matrices of the form

$$
N(p, q, r)=\left(\begin{array}{lll}
0 & 0 & 0 \\
q & 0 & 0 \\
r & p & 0
\end{array}\right),
$$

with horizontal vectors of the form $N(p, q, 0)$. The algebra which they generate is the full Lie algebra, as required. One may also give a direct proof, along the lines of the argument in $\S 9$.

\section{Simple Tate systems}

There is one respect in which the contact system is atypical: it is defined by a single differential form, and (as a result) all maximal integral manifolds are of the same dimension. We shall therefore consider one additional model. Like the contact system it is nil-homogeneous and has a Hodge-theoretic interpretation (from which it draws its name). 
For a purely Lie-theoretic description, consider the group $G_{n}$ of lower triangular $(n+1) \times(n+1)$ unipotent matrices $a=\left(a_{i j}\right)$ with complex entries, where the indices range over the interval $[0, n]$. Let $\omega=a^{-1} d a$ be the MaurerCartan form, and consider the Pfaffian system $\mathscr{I}$ generated by the entries at least two steps below the main diagonal, i.e., by the forms $\omega_{i j}$ for $i>j+1$. These are left-invariant forms which evaluate to $d a_{i j}$ at the identity matrix.

To give a Hodge-theoretic interpretation, use the columns of $a$ to define a decreasing filtration $F^{*}(a)$ of $\mathbb{C}^{n+1}$, namely,

$$
F^{-p}=\operatorname{span}\left\{\alpha_{i} \mid i=0, \ldots, p\right\},
$$

where $\alpha_{i}=e_{i}+a_{i+1, i} e_{i+1}+a_{i+2, i} e_{i+2}+\cdots$. The set $D$ of all such filtrations can be identified with $G$, and the differential system on $G$ just defined agrees with the horizontal system at the identity matrix. Since both systems are homogeneous for $G$ [5, §4], they must agree everywhere. In fact, one may view $D$ as a classifying space (period domain) for mixed Hodge structures whose graded quotients are Tate structures $\mathbb{Z}(p)$ for $p=0, \ldots, n$. Let us say that a mixed Hodge structure whose graded quotients are of the form $\mathbb{Z}(p)^{n_{p}}$ is of Tate type; when the multiplicities $n_{p}$ are one, we shall call the structure simple Tate. Integral manifolds $D$, viewed as variations of mixed Hodge structure, will therefore be called simple Tate variations of genus $n$. Their geometry is controlled by the following result:

(4.1) Theorem. Let $D$ be the period domain for simple Tate variations of genus n. Then:

(i) The dimension $D$ is $n(n+1) / 2$.

(ii) The dimension of the horizontal distribution is $n$.

(iii) The dimension of an integral manifold is at most $\lfloor(n+1) / 2\rfloor$.

(iv) There are integral manifolds of dimension $\lfloor(n+1) / 2\rfloor$.

(v) Any two points $D$ are mutually accessible through the horizontal distribution.

Only the last three assertions require proof. In addition, we have a nondegeneracy result:

(4.2) Definition. A simple Tate variation is nondegenerate if each level of the Hodge filtration is nonconstant.

(4.3) Theorem. A nondegenerate integral manifold of $D$ is of dimension exactly one.

To begin the proof, we require a somewhat more tractable generating set for the differential ideal than the one given by the Maurer-Cartan matrix. The forms obtained using the prescription (3.5) are well suited to our ends. Thus, we find that the coefficient of

$$
e_{0} \wedge \cdots \wedge e_{j+1} \wedge e_{i} \text { for } i>j+1
$$


in

is

$$
d \alpha_{j} \wedge \beta_{-j-1}=d \alpha_{j} \wedge \alpha_{0} \wedge \cdots \wedge \alpha_{j+1}
$$

$$
\phi_{i j}=d a_{i j}-a_{i, j+1} \wedge d a_{j+1, j} \text { for } i>j+1 .
$$

One therefore has the relation

$$
d a_{i j}=a_{i, j+1} \wedge d a_{j+1, j},
$$

where this is taken as an abbreviation for the assertion that $i^{*} \phi_{i j}=0$ for an integral manifold $i: S \rightarrow M$. The new set of generators is not left-invariant. For example, we have $\omega_{30}=d a_{30}-a_{31} d a_{10}-a_{32} d a_{20}+a_{32} a_{21} d a_{10}$, which gives $\omega_{30}=\phi_{30}-a_{32} \phi_{20}$ and $\phi_{30}=\omega_{30}+a_{32} \omega_{20}$.

In any case, we obtain the following:

(4.6) Lemma. For an integral manifold of the simple Tate system we have

(i) $F^{-j}$ is constant if and only if $d a_{j+1, j} \equiv 0$,

(ii) $a_{i j}$ is functionally dependent on $a_{j+1, j}$ for $i>j+1$.

Proof. If $F^{-j}$ is constant, then the coefficient $d \alpha_{j} \wedge \alpha_{0} \wedge \cdots \wedge \alpha_{j}$ in $e_{0} \wedge \cdots \wedge e_{j} \wedge$ $e_{j+1}$, namely, $d a_{j+1, j}$, is zero. The horizontality conditions (4.5) then imply that $d a_{i j}=0$ for all $i>j$. This establishes one half of (i). For the converse, suppose that $d a_{j+1, j}=0$ on an integral manifold $S$. The conditions (4.5) then imply that $d a_{i j}=0$ for all $i>j$, so that $d \alpha_{j}=0$. In the presence of horizontality, this implies that $F^{-j}$ is constant, so that (i) is proved.

For the second part, take the wedge product of (4.5) with $d a_{j+1, j}$ to obtain $d a_{i j} \wedge d a_{j+1, j}=0$, as required.

According to the lemma, the matrix elements just below the diagonal give local parameters, so that an integral manifold of genus $n$ can have dimension no greater than $n$.

Let us improve this bound. For the nondegenerate case, take the exterior derivative of the relation $\phi_{j+2, j}=0$ to get $d a_{j+2, j+1} \wedge d a_{j+1, j}=0$ on $S$. Then the parameters immediately below the diagonal are functionally dependent on the first one, $a_{10}$. This assertion, combined with part (i) of the lemma, implies that the nondegenerate variation is of dimension 1, i.e., is a curve in $D$. Theorem (4.3) is now established.

Degenerate variations of larger dimension do exist. For an example, let $B(t)=\left(\begin{array}{ll}1 & 0 \\ t & 1\end{array}\right)$ and define a block-diagonal matrix

$$
A\left(t_{1}, \ldots, t_{m}\right)=\left(\begin{array}{lll}
B\left(t_{1}\right) & & \\
& \ddots & \\
& & B\left(t_{m}\right)
\end{array}\right)
$$

One verifies that $A(t)$ defines a variation of dimension $m$ and genus $2 m-1$, in accordance with the bounds of the theorem. Note that every other level of 
the Hodge filtration is constant. For an extremal example of odd genus, set

$$
A\left(t_{1}, \ldots, t_{m}\right)=\left(\begin{array}{cccc}
B\left(t_{1}\right) & & & \\
& \ddots & & \\
& & B\left(t_{m}\right) & \\
& & & 1
\end{array}\right)
$$

to get a variation of dimension $m$ and genus $2 m$. These examples establish part (iv) of Theorem (4.1).

Let us now prove, for odd genus $n=2 m-1$, that $\operatorname{dim} S \leq\lfloor(n+1) / 2\rfloor$. To this end, define

$$
\Phi=\bigwedge_{i=0}^{m-1} d \phi_{2 i+1,2 i}= \pm d a_{n, n-1} \wedge d a_{n-1, n-2} \wedge \cdots \wedge d a_{10}
$$

and observe that it is a volume form in the entries on the subdiagonal. Set $\Psi=\Lambda_{l \geq k+2} \phi_{l k}$ and note that

$$
\Psi \wedge \Phi=\bigwedge_{l \geq k+2} d a_{l k} \wedge \Phi= \pm d \mathrm{Vol}
$$

is a volume form in all of the variables. Thus, the Euclidean volume form on $D$ is a product of $m=\lfloor(2 m+1) / 2\rfloor$ forms in $\mathscr{I}^{2}$ with a certain number of forms in $\mathscr{I}^{1}$. By Lemma (2.3), this gives the result.

For even genus $n=2 m$ set

$$
\Phi=\bigwedge_{i=0}^{m-1} d \phi_{2 i+1,2 i}= \pm d a_{n-1, n-2} \wedge d a_{n-2, n-3} \wedge \cdots \wedge d a_{10}
$$

and observe that

$$
\Psi \wedge \Phi \wedge d a_{n, n-1}=\bigwedge_{l \geq k+2} d a_{l k} \wedge \Phi= \pm d \text { Vol }
$$

in a volume form, with $\Psi$ defined as above. Lemma (2.3) applies once again to give the required result. This completes the proof of part (ii) of the theorem.

For part (v) we note that the horizontal tangent space at the identity is given by matrices which are zero except for the subdiagonal entries $a_{i+1, i}$. Since these 'horizontal vectors' generate the full Lie algebra of strictly lower triangular matrices, the hypothesis of Chow's theorem holds at the identity, and, by homogeneity, everywhere, as required.

We conclude with a result on the rigidity of simple Tate variations of maximal dimension:

(4.9) Theorem. Let $S$ be a simple Tate variation of genus $n$ which has maximal dimension $\lfloor(n+1) / 2\rfloor$ and for which $F^{0}$ is nonconstant. Then there is an element $g$ of $G$ such that $S$ is a pull-back of $g A(t)$, where $A(t)$ is given by either (4.7) or (4.8), depending on the parity of $n$.

Proof. We give the proof of $n$ odd, with the even case left as an exercise. Decree that subdiagonal matrix entries $a_{i+1, i}$ be equivalent if they are functionally dependent. Because $d a_{i+2, i+1} \wedge d a_{i+1, i}=0$ on $S$, the equivalence 
classes are unbroken strings of the form $\left\{a_{i+1, i}, a_{i+2, i+1}, \ldots, a_{i+t, i+t-1}\right\}$, with $d a_{i+t, i+t-1}=0$ but $d a_{i+j, i+j-1} \neq 0$ for $j \in[1, t-1]$. Since some subset of the $a_{i+1, i}$ serves as a set of local parameters, there must be precisely $m=\lfloor(n+1) / 2\rfloor$ equivalence classes. Therefore there are precisely $m$ subdiagonal entries $a_{i+1, i}$ with differential zero on $S$, and precisely $m$ with nonzero differential. Moreover, those of zero differential must alternate (in the natural order) with those of nonzero differential. Therefore alternate steps of the Hodge filtration are constant. Left translation by an appropriate group element concludes the argument.

Note the opposition mentioned in the introduction: on the one hand there are rigid and degenerate integral manifolds of rather large dimension; on the other, there are many maximal and nondegenerate integral manifolds of comparatively small dimension. One does not encounter this phenomenon for the contact system.

\section{STRUCTURE OF PERIOD DOMAINS}

We now work towards a proof of Theorem (1.1), beginning with a review of the basic features of classifying spaces for Hodge structures, i.e., the Griffiths period domains $[12,15,17-20]$. Fix a real vector space $H_{\mathbb{R}}$, an integer $w$ (the weight), and a nondegenerate bilinear form $S$ on $H_{\mathrm{R}}$ satisfying $S(x, y)=$ $(-1)^{w} S(y, x)$. A Hodge structure of weight $w$ on $H_{\mathrm{R}}$ is a decomposition

$$
H_{\mathbb{C}}=\bigoplus_{p+q=w} H^{p, q}, \quad \text { where } H^{p, q}=\overline{H^{q, p}} .
$$

This structure is weakly polarized if $H^{p, q}$ is $S$-orthogonal to all $H^{r, s}$ with $(r, s) \neq(q, p)$. Let $h$ be the semilinear form defined by

$$
h(x, y)=(-1)^{w(w-1) / 2} S(x, \bar{y}),
$$

and let $C$ be the Weil operator, defined to act by multiplication by $i^{p-q}$ on $H^{p, q}$. Then $H$ is (strongly) polarized if $h_{C}(x, y)=h(C x, y)$ is positive hermitian.

Let $\check{D}$ denote the set of weakly polarized Hodge structures with fixed Hodge numbers, and let $D$ denote the corresponding subset of strongly polarized structures. Since a point in $\check{D}$ determines a filtration $F^{p}=\bigoplus_{r \geq p} H^{r, s}$ satisfying $\left(F^{p}\right)^{\perp}=F^{w-p}, D$ may be viewed as an algebraic subset of a product of Grassmannians. But $\check{D}$ is homogeneous with respect to the natural action of the special orthogonal group $G_{\mathbb{C}}=\mathrm{SO}(S, \mathbb{C})$, so that it is in fact a complex manifold. The subset $D$ of strongly polarized structures is open and homogeneous for the corresponding real group $G$. Denote by $\mathfrak{g}_{C}$ the Lie algebra of $G_{\mathrm{C}}$, and let $H \in D$ be a reference structure. Then $H$ determines a natural Hodge structure of weight zero on $\mathfrak{g}_{\mathbb{C}}$ [18], where

$$
\mathfrak{g}^{p, q}=\left\{\phi \in \mathfrak{g}_{\mathbb{C}} \mid \phi\left(H^{r, s}\right) \subset H^{r+p, s+q} \text { for all }(r, s)\right\} .
$$


Define subspaces

$$
\mathfrak{g}^{-}=\bigoplus_{p<0} \mathfrak{g}^{p,-p}, \quad \mathfrak{g}^{+}=\bigoplus_{p>0} \mathfrak{g}^{p,-p}, \quad \mathfrak{g}^{0}=\mathfrak{g}^{0,0},
$$

and note that there is a direct sum decomposition $\mathfrak{g}_{\mathbb{C}}=\mathfrak{g}^{-} \oplus \mathfrak{g}^{0} \oplus \mathfrak{g}^{+}$. Because the subspace $\mathfrak{b}=\mathfrak{g}^{0} \oplus \mathfrak{g}^{+}$is the Lie algebra of the isotropy subgroup of the reference structure,

$$
B=\left\{g \in G_{\mathbb{C}} \mid g\left(F^{p}\right) \subset F^{p}\right\},
$$

the complement $\mathfrak{g}^{-}$may be identified with the holomorphic tangent space of $D$ at $H$, and the Hodge component $\mathfrak{g}^{-1,1}$ may be identified with the horizontal subspace:

$$
\mathfrak{g}^{-1,1}=\left\{\phi \mid \phi\left(F^{p}\right) \subset F^{p-1}\right\} .
$$

The Lie bracket is compatible with the Hodge structure on $\mathfrak{g}_{\mathbb{C}},\left[\mathfrak{g}^{p, q}, \mathfrak{g}^{r, s}\right] \subset$ $\mathfrak{g}^{p+r, q+s}$. As we shall see in the next section, $\left[\mathfrak{g}^{-1,1}, \mathfrak{g}^{-1,1}\right]=\mathfrak{g}^{-2,2}$, so that the horizontal distribution is not integrable, provided that $\mathfrak{g}^{-2,2} \neq 0$. In the weight two case $\mathfrak{g}^{-2,2} \neq 0$ if and only if $h^{2,0}>1$.

\section{Hodge fRAMES}

A Hodge frame [10] for $H$ is a set of ordered bases $B^{p, q}$ for $H^{p, q}$ such that

(i) $B^{p, q}$ is an $h_{C}$-unitary basis for $H^{p, q}$.

(ii) $B^{p, q}=\overline{B^{q, p}}$.

The matrix of $S$ has a natural block decomposition relative to a Hodge frame, with all blocks zero except for those on the anti-diagonal, which are, up to sign, identity matrices. Thus, in the weight two case,

$$
S=\left(\begin{array}{ccc}
0 & 0 & I_{a} \\
0 & -I_{b} & 0 \\
I_{a} & 0 & 0
\end{array}\right)
$$

Here we have (as we shall henceforth) written $a$ for $h^{2,0}$ and $b$ for $h^{1,1}$. Elements of $\mathfrak{g}_{\mathbb{C}}$ also have a block decomposition, where

(i) elements in $\mathfrak{g}^{-}$are strictly block lower triangular,

(ii) elements in $\mathfrak{g}^{-1,1}$ have nonzero blocks only in positions immediately below the main diagonal.

Thus, in the weight two case we have, for $N \in \mathfrak{g}^{-}$,

$$
N=\left(\begin{array}{lll}
0 & 0 & 0 \\
X & 0 & 0 \\
Y & Z & 0
\end{array}\right)
$$

The condition that the matrix $N$ be in the orthogonal Lie algebra, ${ }^{T} N S+S N=$ 0 , imposes additional restrictions, namely $Z={ }^{T} X$ and ${ }^{T} Y=-Y$ so that

$$
N=N(X, Y)=\left(\begin{array}{ccc}
0 & 0 & 0 \\
X & 0 & 0 \\
Y & T^{X} X & 0
\end{array}\right)
$$


It follows that $D$ has dimension $a b+\frac{1}{2} a(a-1)$, since the tangent vector $N(X, Y)$ depends on that many parameters. Because horizontal tangent vectors have the form $N(X, 0)$, the horizontal tangent distribution has dimension $a b$. The Lie bracket is given by

$$
\left[N\left(X_{1}, Y_{1}\right), N\left(X_{2}, Y_{2}\right)\right]=N\left(0,{ }^{T} X_{1} X_{2}-{ }^{T} X_{2} X_{1}\right),
$$

so that if $E_{i j}$ denotes a matrix with a single nonzero entry, namely, a 1 in position $(i, j)$, then

$$
\left[N\left(E_{i j}, 0\right), N\left(E_{k l}, 0\right)\right]=N\left(0, \delta_{i k}\left(E_{j l}-E_{l j}\right)\right),
$$

where $\delta_{i k}$ is Kronecker's delta. Therefore $\left[\mathfrak{g}^{-1,1}, \mathfrak{g}^{-1,1}\right]=\mathfrak{g}^{-2,2}$, so that the horizontal distribution is nonintegrable, provided that $\mathfrak{g}^{-2,2} \neq 0$. Since $Y$ is antisymmetric, this last condition is equivalent to $h^{2,0}>1$.

\section{CANonical coordinates}

Consider now the map from the nilpotent subalgebra $\mathfrak{g}^{-}$to $\check{D}$ defined by applying $\exp N$ to a reference structure $H \in D$. Since $D$ is open in $\check{D}$, the image point will be in $D$ if the norm of $N$ is sufficiently small. Moreover, the differential of this map is (by construction) the identity, so that $N \mapsto(\exp N) \cdot H$ gives local coordinates at $H$. Thus $D$ is locally homogeneous for a unipotent group in a natural way. Let us consider these coordinates in detail for the weight two case. Write the exponential map as

$$
(X, Y) \mapsto(\exp N(X, Y)) \cdot H,
$$

so that the components of the matrices $X$ and $Y$ give the local coordiantes. At the origin of this system the horizontal distribution is defined by $d Y=0$. Since the horizontal distribution is homogeneous, it is defined at nearby points by the vanishing of the left-invariant extension of $d Y$. An explicit formula is given by the next result:

(7.1) Lemma. The left-invariant extension of $d Y$ at the origin is the matrixvalued form

$$
\omega=d Y+\frac{1}{2}\left({ }^{T} d X \cdot X-{ }^{T} X \cdot d X\right) .
$$

Proof. The left-invariant extension of $d Y$ is given by the block in the lower left corner of the Maurer-Cartan matrix $\Omega=e^{-N} d e^{N}$. For the exponential we have

$$
e^{N}=\left(\begin{array}{ccc}
1 & 0 & 0 \\
X & 1 & 0 \\
f(X, Y) & { }^{T} X & 1
\end{array}\right),
$$

where $f(X, Y)=Y+\frac{1}{2}^{T} X \cdot X$. The Maurer-Cartan form is then

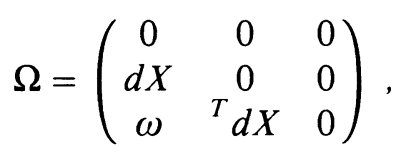


where

$$
\omega=d f(X, Y)-{ }^{T} X d X=d Y+\frac{1}{2}\left({ }^{T} d X \cdot X-{ }^{T} X \cdot d X\right) .
$$

Thus $\omega$ has the required form.

(7.2) Remark. The analogy with the contact system becomes perfect in a slightly different frame of reference. In $\S 3$ we used the coefficients of

$$
U=\left(\begin{array}{lll}
1 & 0 & 0 \\
q & 1 & 0 \\
r & p & 1
\end{array}\right)
$$

as coordinates. Instead, let us write $U=e^{N}$, with

$$
N=\left(\begin{array}{lll}
0 & 0 & 0 \\
Q & 0 & 0 \\
R & P & 0
\end{array}\right)
$$

and use the matrix entries of $N$ as coordinates. The Maurer-Cartan form then becomes

$$
\Omega=\left(\begin{array}{ccc}
0 & 0 & 0 \\
d Q & 0 & 0 \\
\omega & d P & 0
\end{array}\right)
$$

where $\omega=d R+\frac{1}{2}(d P \cdot Q-P \cdot d Q)$ and the dot denotes matrix product. Thus, if we require that $P={ }^{T} Q$ and that $R$ be skew-symmetric, then we obtain the matrix-valued form of Lemma (7.1).

\section{Proof of the MAIN THEOREM}

We now exploit the properties of the matrix-valued contact form $\omega$ to establish the bound of Theorem (1.1):

$$
\operatorname{dim} S \leq \frac{1}{2} h^{2,0} h^{1,1} \text { for } h^{2,0}>1 .
$$

Again we write $a$ for $h^{2,0}$ and $b$ for $h^{1,1}$. We first give a proof for $a=2 p$ even. To this end, note that the components of $\omega$ can be written explicitly as

$$
\omega_{i j}=d Y_{i j}+\frac{1}{2} \sum_{t=1}^{b}\left[d X_{t i} \cdot X_{t j}-X_{t i} \cdot d X_{t j}\right]
$$

Define a 'volume form in the $Y_{i j}$ variables' by $d Y_{J_{1}} \wedge \cdots \wedge d Y_{J_{c}}$, where $\left(J_{1}, \ldots, J_{c}\right)$ is an ordering of the set $\{(i, j) \mid 1 \leq i<j \leq a\}$. Since the $X_{i j}$ vanish at the origin of the coordinate system, the form

$$
\Phi=\omega_{J_{1}} \wedge \cdots \wedge \omega_{J_{c}}
$$

satisfies

$$
\Phi=d Y_{J_{1}} \wedge \cdots \wedge d Y_{J_{c}}
$$

there. Consequently the partial volume form $\Phi$ is a volume form in the $Y_{i j}$ for $Y$ small. Next, observe that

$$
d \omega_{i j}=-\sum_{t=1}^{b} d X_{t i} \wedge d X_{t j}
$$


so that the expression

$$
\Psi=d \omega_{12}+d \omega_{34}+\cdots+d \omega_{2 p-1,2 p}
$$

defines a nondegenerate two-form in the variables $X_{i j}$. Therefore $\Psi^{p b}$ is a volume form in the $X_{i j}$, from which it follows that the product $\Omega=\Phi \wedge \Psi^{p b}$ defines a volume form at the origin (and hence locally) in all the variables. Since $\Phi$ and $\Psi^{p b}$ are products of one-forms and of two-forms in the differential ideal of the horizontal distribution, respectively, Lemma (2.3) applies to give

$$
\operatorname{dim} S \leq p b=\frac{1}{2} a b=\frac{1}{2} h^{2,0} h^{1,1},
$$

as required.

We now study the odd case, with $h^{2,0}=2 p+1$ in force for the rest of this section. A crude inequality is easily obtained:

(8.1) Lemma. If $h^{2,0}=2 p+1$ is odd then $\operatorname{dim} S \leq p b+b$.

Proof. Apply Lemma (2.3) to the volume form

$$
\Omega=\Phi \wedge \Psi^{p b} \wedge\left(d X_{1,2 p+1} \wedge \cdots \wedge d X_{b, 2 p+1}\right) .
$$

Since the required bound is

$$
\operatorname{dim} S \leq \frac{1}{2} h^{2,0} h^{1,1}=\frac{1}{2} a b=p b+\frac{1}{2} b,
$$

we must improve the inequality above by $\frac{1}{2} b$ units. The presence of additional one-forms in the differential ideal of an integral submanifold $S$ allows one to do this. To make a precise statement, let $I(S)$ be the differential ideal of germs of forms whose pullback to $S$ vanishes, and note that $I(\omega) \mid S$ is contained in $I(S)$.

(8.2) Lemma. Suppose that there are one-forms $\eta_{1}, \ldots, \eta_{e}$ in the ideal $I(S)$ such that

$$
\Omega=\Phi \wedge \Psi^{p b} \wedge\left(\eta_{1} \wedge \cdots \wedge \eta_{e}\right) \wedge\left(d X_{e+1,2 p+1} \wedge \cdots \wedge d X_{b, 2 p+1}\right)
$$

is a volume form. Then $\operatorname{dim} S \leq p b+(b-e)$.

Proof. Apply Lemma (2.3).

To produce these additional one-forms, we establish the following two results:

(8.3) Lemma. Let $V$ be an $r$-dimensional space of $b \times a$ matrices. Let $e_{k}$ denote the $k$ th standard unit vector, and let $C_{k}$ be the dimension of the vector space $V\left(e_{k}\right)=\left\{A e_{k} \mid A \in V\right\}$. Then $\max C_{k} \geq r / a$.

Proof. Let $\left(A^{1}, \ldots, A^{r}\right)$ be an ordered basis for $V$. Let $\hat{A}^{i}$ denote the row vector obtained by setting the rows of $A^{i}$ end-to-end in the natural order, and let $\mathscr{A}$ be the matrix whose rows are the $\hat{A}^{i}$. Apply Gauss-Jordan reduction to $\mathscr{A}$ to obtain an echelon matrix $\mathscr{B}$ with rows $\widehat{B}^{i}$, and let $\left(B^{1}, \ldots, B^{r}\right)$ be the 
corresponding ordered basis of $V$. Let $\widehat{L}(k)$ be the leading nonzero entry in $\widehat{B}^{k}$, and let $L(k)=\left(i_{k}, j_{k}\right)$ be the corresponding index in $B^{k}$, where

$$
B_{L(k)}^{k}=1, \quad B_{L(k)}^{s}=0 \quad \text { for } s \neq k
$$

Let $C_{k}$ be the number of $B_{i}$ for which a leading entry occurs in column $k$. If $\max C_{k}<r / a$ then $C_{1}+\cdots+C_{a}<r$. But $\operatorname{dim} V=C_{1}+\cdots+C_{a}$, so this is a contradiction.

(8.4) Remark. Here is an invariant formulation: Let $U$ and $W$ be vector spaces of dimension $a$ and $b$, respectively. Let $V$ be an $r$-dimensional subspace of $\operatorname{Hom}(U, W)$, let $u$ be an element of $U$, and consider the vector space $V(u)$. Then $\operatorname{dim} V(u) \geq r / a$ for some $u \in U$.

(8.5) Lemma. Suppose that $\operatorname{dim} S \leq p b+e$. Then there exist at least

$$
m=\lceil(p b+b-e) /(2 p+1)\rceil
$$

one-forms $\eta_{1}, \ldots, \eta_{m}$ vanishing on $S$ which are independent modulo the $d Y_{j k}$ and which satisfy the following condition: if

$$
\eta_{i}=\sum_{j, k} A_{j k}^{i} d X_{j k}+\text { terms in the } d Y_{r s},
$$

then there is a column index $c \in[1, a]$, a subset of row indices, $K \subset[1, r]$, and a function $r:[1, m] \rightarrow K$ such that

$$
A_{r(c), c}^{i}=1, \quad A_{j c}^{i}=0 \quad \text { for } j \in K \text { but } j \neq r(i) .
$$

Here $[x]$ denotes the least integer $n$ such that $n \geq x$.

Proof. Since $\operatorname{dim} D=a b+a(a-1) / 2=(2 p+1) b+a(a-1) / 2$, the tangent space to $S$ at the origin is defined by at least $p b+(b-e)+a(a-1) / 2$ independent one-forms, all in the differential ideal of the horizontal distribution. Of these, $a(a-1) / 2$ may be taken to be the forms $d Y_{r s}$. Consequently there are at least $k=p b+(b-e)$ forms $\alpha_{1}, \ldots, \alpha_{k}$ which are independent modulo the $d Y_{r s}$. Lemma (8.3) guarantees the existence of independent forms $\eta_{1}, \ldots, \eta_{m}$ in the span of the $\alpha$ 's with $m$ as above, satisfying the stated conditions on the coefficient matrices.

(8.6) Remark. After a permutation of the rows and columns of $X$ we may assume that $c=2 p+1, K=[1, m]$, and $r=\mathrm{id}$, so that we are in the situation of Lemma (8.2).

(8.7) Lemma. If $\operatorname{dim} S \leq p b+e$ then $\operatorname{dim} S \leq p b+\lfloor(p b+e) /(2 p+1)\rfloor$, where $\lfloor x\rfloor$ is the greatest integer less than or equal to $x$.

Proof. Combine Lemmas (8.2) and (8.5).

With these results in hand it is already possible to improve the inequality of Lemma (8.1): 
(8.8) Corollary. If $h^{2,0}=2 p+1$ is odd, then

$$
\operatorname{dim} S \leq p b+\lfloor b(p+1) /(2 p+1)\rfloor \approx p b+b / 2 .
$$

Proof. Combine Lemmas (8.1) and (8.7).

The bound given by the corollary is much closer to what is required, since $p b+b / 2=h^{2,0} h^{1,1} / 2$. To complete the proof of the main theorem we show that the process described above can be applied repeatedly to get the required inequality. To this end, set $d_{i}=p b+e_{i}$ and suppose that the bound $\operatorname{dim} S \leq d_{i}$ is established. Then Lemma (8.7) implies that $\operatorname{dim} S \leq d_{i+1}$ with

$$
e_{i+1}=\left\lfloor\left(p b+e_{i}\right) /(2 p+1)\right\rfloor .
$$

It is enough to show that the integers $e_{i}$ form a strictly decreasing sequence so long as $e_{i}>b / 2: e_{i+1}<e_{i}$ if $e_{i}>b / 2$. To do this, observe that

$$
\begin{aligned}
e_{i+1}-e_{i} & =\left\lfloor\left(p b+e_{i}\right) /(2 p+1)\right\rfloor-e_{i} \\
& =\left\lfloor\left(p b+e_{i}\right) /(2 p+1)-e_{i}\right\rfloor, \\
& =\left\lfloor\left(p b-2 p e_{i}\right) /(2 p+1)\right\rfloor,
\end{aligned}
$$

which is negative if $e_{i}>b / 2$, as required.

Remarks (8.9). (i) As in the case of simple Tate variations, there is a rigidity theorem [2, Theorem 6.3 and Remark 6.6]. In brief, whenever $a=h^{2,0}>2$ and $b=h^{1,1}$ is even, then every integral manifold of dimension $\frac{1}{2} a b$ in $D$ prolongs to one which is homogeneous for an action of a copy of $\mathrm{SU}(p, q)$ imbedded in the isometry group of $D$, where $p=a$ and $q=b / 2$. Moreover, any two such integral manifolds are conjugate under the isometry group. When $h^{2,0}=2$, however, rigidity fails [2, Remark 5.5.c].

(ii) Again as in the case of Tate variations, there are maximal integral manifolds which are not of maximal dimension. The main result of [8] shows that almost all hypersurfaces of dimension greater than $n>1$ define maximal integral manifolds via $X \mapsto H_{0}^{n}(X)$, where the subscript denotes primitive cohomology. All surfaces of degree $d$ satisfy the hypotheses of [8], although this is of interest only for $d>4$. For these, the dimension of $S$ is $O\left(d^{3}\right)$, while $\frac{1}{2} h^{2,0} h^{1,1}$ is $O\left(d^{6}\right)$. On the other hand, hypersurface variations satisfy nondegeneracy conditions of various kinds, e.g., $F^{k}$ has no fixed subspaces, in contrast to what happens for the $\mathrm{SU}(p, q)$-homogeneous variations mentioned above. As yet there is no good understanding of the interplay between nondegeneracy conditions, geometry, and dimension restrictions.

\section{ACCESSIBILITY}

We now turn to the accessibility properties of period domains:

(9.1) Theorem. Let $H_{0}$ be a point of $D$. Then there is a neighborhood $\mathscr{U}$ of $H_{0}$ consisting entirely of structures accessible from $H_{0}$.

This result follows from the theorem of Chow [11] mentioned in $\S 2$, provided that we verify its hypothesis: that horizontal vector fields generate the 
full tangent space under Lie bracket. The holomorphic tangent space of a period domain is given by $\mathfrak{g}^{-}$, where in the weight two case $\mathfrak{g}^{-}=\mathfrak{g}^{-1,1} \oplus \mathfrak{g}^{-2,2}$, and, according to the last formula of $\S 6,\left[\mathfrak{g}^{-1,1}, \mathfrak{g}^{-1,1}\right]=\mathfrak{g}^{-2,2}$, so that Chow's theorem applies.

We also give a proof using an explicit computation in canonical coordinates $(X, Y)$. Note first that a curve $\gamma(t)$ of the form $Y=Y_{0}, X=P t$, where $Y_{0}$ and $P$ are constant matrices, is horizontal. Therefore all points of the form $(X, 0)$, for $X$ sufficiently small, are accessible from the origin. To reach other points we shall construct lifts of suitable piecewise linear paths in the set of points with $Y=0$. To this end we define a bracket operator by $[P, Q]=$ ${ }^{T} P Q-{ }^{T} Q P$ and note the following:

(9.2) Lemma. Let $\mathscr{U}$ be a canonical coordinate neighborhood, let $\mathscr{U}_{0}$ be the set defined by projection of $\mathscr{U}$ along $(X, Y) \mapsto X$, and let $P, Q \in \mathscr{U}_{0}$. Then $(Q, c+[P, Q] / 2)$ is accessible from $(0, c)$.

Proof. Define paths $\alpha, \beta$, and $\gamma$ in the canonical coordinate neighborhood by $\alpha(t)=(P t, c), \beta(t)=(P+t(Q-P), Y(t))$ and $\gamma(t)=((1-t) Q, Y(1))$, where $t \in[0,1]$. Choose the function $Y(t)$ so that $\beta$ is horizontal with initial condition $Y(0)=c$, and note that $\alpha$ and $\gamma$ are horizontal by construction. Then

$$
Y(1)-Y(0)=\int_{\beta} d Y=\int_{\alpha+\beta+\gamma} d Y=\int_{\alpha+\beta+\gamma}\left({ }^{T} X \cdot d X-{ }^{T} d X \cdot X\right) / 2 .
$$

Consider the two-chain defined by $R(s, t)=P s+Q t$, where $(s, t)$ is in the standard two-simplex $\Delta$, defined by $s \geq 0, t \geq 0$, and $s+t \leq 1$. Then

$$
\int_{\alpha+\beta+\gamma}\left({ }^{T} X \cdot d X-{ }^{T} d X \cdot X\right) / 2=\int_{\partial R}\left({ }^{T} X \cdot d X-{ }^{T} d X \cdot X\right) / 2,
$$

so that by Stokes' theorem one has

$$
Y(1)-Y(0)=\int_{\Delta}{ }^{T} d X \wedge d X=\int_{\Delta}[P, Q] d s \wedge d t=[P, Q] / 2 .
$$

Therefore $\alpha+\beta$ joins $(0, c)$ to $(Q, c+[P, Q] / 2)$, as required.

To complete the proof, fix a point $(R, S)$ in $\mathscr{U}$ and let $X_{i}, Y_{i} \in \mathfrak{g}^{-1,1}$ be elements such that

$$
2 S=\sum_{i=1}^{n}\left[X_{i}, Y_{i}\right]
$$

This is possible because $\left[\mathfrak{g}^{-1,1}, \mathfrak{g}^{-1,1}\right]=\mathfrak{g}^{-2,2}$. Let $P_{1}, \ldots, P_{2 n+1}$ be elements of $\mathfrak{g}^{-1,1}$ such that $P_{2 n+1}=R$ and

$$
\sum_{i=1}^{2 n}\left[P_{i}, P_{i+1}\right]=\sum_{i=1}^{n}\left[X_{i}, Y_{i}\right]
$$

One can always write the $2 n+1$ elements $P_{i}$ in terms of the $2 n+1$ elements $R, X_{i}$, and $Y_{i}$ in order to achieve this. Let $\gamma:[0,1] \longrightarrow \mathscr{U}$ be a path in the 
domain $Y=0$ which joins the sequence of points $\left(0, P_{1}, \ldots, P_{2 n+1}\right)$ in the given order, and let $\tilde{\gamma}$ be the horizontal lift with left endpoint at $(0,0)$. By repeated applications of Lemma (9.2) one calculates the right endpoint to be $(R, S)$. Therefore all points in $\mathscr{U}$ are accessible from the origin, as required.

An immediate corollary of Theorem (9.1) is the following:

(9.3) Theorem. Let $H_{0}$ be a point of $D$. Then all points of $D$ are accessible from $H_{0}$.

Proof. Let $H_{1}$ be another point of $D$. Join $H_{0}$ to $H_{1}$ by a curve $\gamma$. By compactness there is a cover of $\gamma$ by neighborhoods consisting of mutually accessible points, from which the result follows.

\section{APPENDIX: GeOMETRIC LeGENDRE MANIFOLDS}

In this appendix we give an example of a geometric 4-dimensional Legendre manifold. To this end, let each of $A$ and $B$ consist of three lines in general position in $\mathbb{P}^{2}$, with $A \cup B$ in general position as well. Consider the mixed Hodge structure

$$
H(A, B)=H^{2}\left(\mathbb{P}^{2}-B, A-B\right),
$$

with an appropriate shift of weights (tensor with $\mathbb{Z}(-2)$ ). We claim (1) that $H(A, B)$ is a Heisenberg structure of genus 4 , and (2) that $(A, B) \mapsto H(A, B)$ is a variation of dimension 4. The argument depends on an analysis of the extension of mixed Hodge structures [3-5] defined by

$$
0 \rightarrow W_{0} \rightarrow W_{2} \rightarrow \mathrm{Gr}_{2}^{W} \rightarrow 0 \text {. }
$$

We show below that the extension is given geometrically by the cross-ratios of the points which $B$ cuts out on $A$ with respect to the double points of $A$. These cross-ratios give four independent moduli, so that $H(A, B)$ depends on at least that many. By the bound of Theorem (3.3), $H(A, B)$ depends on precisely four parameters. To verify the above assertions, consider the cohomology sequence of the pair $\left(\mathbb{P}^{2}-B, A-B\right)$ :

$$
H^{1}\left(\mathbb{P}^{2}-B\right) \stackrel{i^{*}}{\longrightarrow} H^{1}(A-B) \stackrel{\delta}{\longrightarrow} H^{2}\left(\mathbb{P}^{2}-B, A-B\right) \stackrel{j^{*}}{\longrightarrow} H^{2}\left(\mathbb{P}^{2}-B\right) .
$$

The right-hand group is isomorphic to $H^{2}\left(\mathbb{C}^{* 2}\right) \cong \mathbb{Z}(-2)$, with canonical generator $d z / z \wedge d w / w$ of weight 4 , and the left-hand group is isomorphic to $H^{1}\left(\mathbb{C}^{* 2}\right) \cong \mathbb{Z}(-1) \oplus \mathbb{Z}(-1)$, with canonical generators $d z / z$ and $d w / w$ of weight 2 . The weight 2 part of the relative cohomology can be identified with the image of $\delta$, i.e., with the cokernel of $i^{*}$. To calculate the term $H^{1}(A-B)$, set

$$
A=\bigcup_{i=0}^{2} A_{i}, \quad B=\bigcup_{i=0}^{2} B_{i},
$$


let $A_{i}^{\prime}=A_{i}-B \cong \mathbb{C}-\{0,1\}$, and let $D$ be the set of double points of $A$. The Mayer-Vietoris spectral sequence gives an exact sequence

$$
\bigoplus_{i=0}^{2} H^{0}\left(A_{i}^{\prime}\right) \rightarrow H^{0}(D) \rightarrow H^{1}(A-B) \rightarrow \bigoplus_{i=0}^{2} H^{1}\left(A_{i}^{\prime}\right) \rightarrow 0,
$$

from which one finds the weight 2 part of $H^{1}(A-B)$ is 6-dimensional and that the weight 0 part is 1 -dimensional. The weight 2 part of $H(A, B)$ is $H^{1}(A-B)$ modulo the image of $i^{*}$; one concludes that it is of dimension 1 in weight 0 and of dimension 4 in weight 2 . Now let $\left\{A_{i} \cdot A_{j} \mid 0 \leq i<j \leq 2\right\}$ be the set of double points, let $\left\{A_{i} \cdot B_{j} \mid 0 \leq i, j \leq 2\right\}$ be the set of punctures, and let $\omega_{i}\left(B_{j}-B_{k}\right)$ be the unique meromorphic differential on $A-B$ supported on $A_{i}$ with residue cycle $2 \pi i A_{i} \cdot\left(B_{j}-B_{k}\right)$. There are nine of these, subject to the relations below:

$$
\begin{gathered}
\sum_{i<j}(-1)^{(i+j)} \omega_{k}\left(B_{i}-B_{j}\right)=0 \\
\sum_{k} \omega_{k}\left(B_{i}-B_{j}\right) \equiv 0 \text { modulo the image of } i^{*}
\end{gathered}
$$

Their cohomology classes generate the integral lattice of $\mathrm{Gr}_{2}^{W} H(A, B) \cong$ $H^{1}(A-B) /$ image $\left(i^{*}\right)$.

Let $\delta$ be a generator for the weight zero part of the first homology of $A-B$. From the homology sequence dual to (10.2), one sees that $\delta$ is given by a sum of oriented paths $\delta_{i}$, each supported in $A_{i}^{\prime}$ and connecting the two nodes of $A$ which lie in that component. According to the results of [3-5], the extension of mixed Hodge structures associated to $W_{2} H(A, B)$ is given by the integrals $\int_{\delta} \omega_{i}\left(B_{j}-B_{k}\right)$. But this integral determines a natural cross-ratio:

$$
\exp \left(2 \pi i \int_{\delta} \omega_{i}\left(B_{j}-B_{k}\right)\right)=\operatorname{cross}-\operatorname{ratio}\left(A_{i} \cdot B_{j}, A_{i} \cdot A_{i}^{\prime}, A_{i} \cdot B_{k}, A_{i} \cdot A_{i}^{\prime \prime}\right),
$$

where cross-ratio $(0,1, \infty, a)=a$ and $\partial \delta_{i}=A_{i} \cdot A_{i}^{\prime \prime}-A_{i} \cdot A_{i}^{\prime}$. The geometric data for these integrals is therefore a 5-tuple of points on each component $\mathbb{P}^{1}$ of $A$, with two points of each 5-tuple distinguished by the fact that they are nodes rather than punctures. A 5-tuple in $\mathbb{P}^{1}$ depends on two moduli, for a total of six parameters for the integrals. However, the parameters are not independent, since the punctures occur in collinear triples. This imposes two relations, given (for example) by the fact that the periods of $i^{*} d z / z$ and $i^{*} d w / w$ vanish on $\delta$. For a formal proof of this last fact, observe that both differentials map to zero in the relative cohomology group in (10.1). This leaves four independent parameters for the integrals, and hence for the mixed Hodge structure $H^{1}(A-B) /$ image $\left(i^{*}\right) \cong W_{2} H^{2}\left(\mathbb{P}^{2}-B, A-B\right)$, as required. 


\section{BIBLIOGRAPHY}

1. V. I. Arnold, Mathematical methods of classical mechanics, Springer-Verlag, 1978, pp. 462.

2. J. A. Carlson, Bounds on the dimension of a variation of Hodge structure, Trans. Amer. Math. Soc. 294 (1986), 45-64.

3. __ The obstruction to splitting a mixed Hodge structure over the integers. I, Univ. of Utah, preprint, 1979.

4. __ Extensions of mixed Hodge structures, Journées de Géométrie Algébrique d'Angers 1979, Sijthoff \& Noordhoff, Alphen aan den Rijn, The Netherlands, 1980, pp. 107-127.

5. _ The geometry of the extension class of a mixed Hodge structure, Proc. Sympos. Pure Math., vol. 46, Amer. Math. Soc., Providence, R. I., 1987, pp. 199-222.

6. J. A. Carlson, M. L. Green, P. A. Griffiths and J. Harris, Infinitesimal variations of Hodge structures. I, Compositio Math. 50 (1983), 109-205.

7. J. A. Carlson and P. A. Griffiths, Infinitesimal variations of Hodge structure and the global Torelli problem, Journées de Géométrie Algébrique d'Angers 1979, Sijthoff \& Noordhoff, Alphen aan den Rijn, The Netherlands, 1980, pp. 51-76.

8. J. A. Carlson and Ron Donagi, Hypersurface variations are maximal, Invent. Math. 89 (1987), 371-374.

9. J. A. Carlson and C. Simpson, Shimura varieties of weight two Hodge structures, Hodge Theory, Lecture Notes in Math., vol. 1246, Springer-Verlag, 1987, pp. 1-15.

10. M. Cornalba and P. A. Griffiths, Some transcendental aspects of algebraic geometry, Proc. Sympos. Pure Math., vol. 24, Amer. Math. Soc., Providence, R. I., 1975, pp. 3-110.

11. W. L. Chow, Über Systeme von linearen partiellen Differentialgleichungen erster Ordnung, Math. Ann. 117 (1939), 98-105. MR 1, 313.

12. P. Deligne, Travaux de Griffiths, Séminaire Bourbaki 376, Hermann, Paris, 1970.

13. _ _ Théorie d'Hodge. II, Inst. Hautes Études Sci. Publ. Math. 40 (1971), (1971), 5-58.

14. __ Théorie d'Hodge. III, Inst. Hautes Études Sci. Publ. Math. 44 (1975), 5-77.

15. P. A. Griffiths, On the periods of certain rational integrals. I, Amer. J. Math. 90 (1968), 569625.

16. __ Periods of rational integrals, I, II, Ann. of Math. (2) 90 (1969), 460-526 and 805-865.

17. __ Periods of integrals on algebraic manifolds. III, Inst. Hautes Études Sci. Publ. Math. 38 (1970), 125-180.

18. P. A. Griffiths and W. Schmid, Recent developments on Hodge theory: a discussion of techniques and results, Proc. Bombay Colloq. Discrete Subgroups of Lie Groups, Tata Institute for Fundamental Research, Bombay, 1973.

19. __ Locally homogeneous complex manifolds, Acta Math. 79 (1964), 109-326.

20. P. A. Griffiths and L. Tu, Variation of Hodge structure, Topics in Transcendental Algebraic Geometry (P. A. Griffiths, ed.), Ann. of Math. Studies, no. 106, Princeton Univ. Press, 1984.

21. R. Hermann, On the accessibility problem in control theory, Internat. Sympos. Nonlinear Differential Equations and Nonlinear Mechanics, Academic Press, New York, 1963, pp. 325-332. MR 26 \#6891.

22. H. J. Sussman, Orbits of families of vector fields and integrability of distributions, Trans. Amer. Math. Soc. 180 (1973), 171-188.

23. S. Usui, Variations of mixed Hodge structure arising from family of logarithmic deformations, Ann. Sci. École Norm. Sup. 16 (1983), 91-107; II: Classifying space, Duke Math. J. 51 (1984), 851-857.

Department of Mathematics, University of Utah, Salt Lake City, Utah 84112 